\title{
Enseignements tirés du traitement du remblai gagné sur la mer - Marina J. Khoury (Liban)
}

\section{J.-B. ESTA}

Professeur à l'École supérieure d'ingénieurs

de Beyrouth Inmeuble TRAD rue Issa-Maalouf Beyrouth, Libars

\section{A. EL KHOURY}

DEA Mécanigue des sols ESIB Sols renforcés

Beyrouth, Liban

\section{S. FATTOUH}

B.S. Geology American University of Beirut Soil mechanics associates Beyrouth, Liban
Un remblai constitué par un sable dragué en mer au large des côtes libanaises a été gagné sur la mer entre Antelias et Dbaye au Nord de Beytouth sur une superficie d'un million et cent mille mètres carrés. Certaines parcelles entourant la Marina ont été traitées par vibro-compactage et pilonnage intensif pour réduire - voire annuler - le risque de liquéfaction et éviter, sous chargement statique, des tassements totaux et differentiels importants. Larticle présente les méthodes de contrôle du traitement choisj et montre, pour deux immeubles de 12 étages construits sur radier" sur ce sol amélioré, une bonne concordance entre les tassements mesurés et les tassements calculés par la methode pressiometrique, les modules de déformation Ménard étant obtenus par corrélation à partir d'essais pénétrométriques statiques ANDINA.

Mots-clés : remblai, amélioration des sols, vibrocompactage, pilonnage, liquéfaction, tassement.

\section{Lessons drawn out of the treatment of the reclaimed sea-land fill - J. Khoury Marina (Lebanon)}

A sea sand fill dredged off the lebarese coasts was dumped to reclaim sea-land between Antelias and Dbayé, North of Bejut covering an area of one million and one hundred thousand squared meters. Some plots surrounding the Marina have been treated by vibro-compaction and dynamic compaction to reduce, if not cancel, the risk of liquefaction and to avoid considerable total anc differential settlements under static loak. The aricle displays the chosen verfication methods of improvement and shows, for two 12-storeys buildngs erected on a raft over this treated ground, a very good conformity between measured settlements and setternents conputed by pressuremeiric method, the Menard deformation noduli being obtained by correlation starting from ANDINA static penetrometer tests. 


\section{Introduction}

Commencè en 1982, le remblaiement du terre-plein gagné sur la mer entre Antelias et Dbayé au nord de Beyrouth s'est poursuivi malgré la guerre et a été achevé en 1995. Couvrant une surface proche d'un million cent mille mètres carrés, le remblai mis en place par l'entreprise SNE Joseph Khoury est protégé, côté mer, par des enrochements et par des acropodes (Fig. 1). Mise à part la zone entourant la Marina où des enrochements et un tout-venant calcaire ont été mis en place, le remblai est constitué par un sable dragué en mer au large des côtes libanaises et déversé hydrauliquement par l'entreprise hollandaise HAM.

Afin de faciliter les travaux de construction futurs et eu égard aux règles de l'urbanisme en vigueur au Liban, ce remblaí a été mis en place grossièrement à la cote $+1,3$ alors que les routes desservant les parcelles sont à la cote + 8 environ, ce quí permet dès le départ de prévoir au moins deux niveaux de sous-sols pour les immeubles à construire.

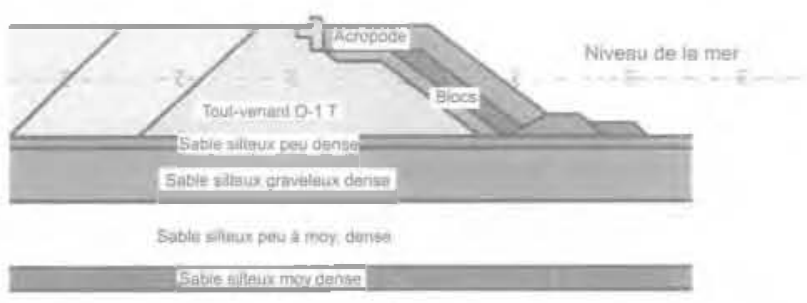

Substratum rocheus

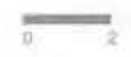

Echele 11no

10

คG. 1 Coupe de la digue. Terrain gagne sur la mer. Barrier section. Heclairned sea-land.

\section{Nature du remblai utilisé}

\section{1}

\section{Propriétés physiques}

Les analyses faites au laboratoire de l'École d'ingénieurs de Beyrouth (ESIB) montrent que le sable utilisé est un sable calcaire dont la composition chimique est la suivante.

\begin{tabular}{lc}
\hline Perte au feu & $43,85 \%$ \\
\hline Chaux & $48,40 \%$ \\
\hline Magnésie $(\mathrm{MgO})$ & $3,62 \%$ \\
\hline Silice $\left(\mathrm{SO}_{2}\right)$ & $3,70 \%$ \\
\hline Alumine et oxyde ferrique $\left(\mathrm{Fe}_{2} \mathrm{O}_{2}+\mathrm{Al}_{2} \mathrm{O}_{3}\right)$ & $0,60 \%$ \\
\hline Autres & $0,43 \%$ \\
\hline
\end{tabular}

Ce sable proviendrait des grès dunaires wümiens qui affleurent le long du rivage [Sanlaville, 1977). Par ailleurs, toutes les analyses granulometriques montrent qu'il s'agit d'un sable graveleux dont le diamètre est compris entre 10 et $0,1 \mathrm{~mm}$, le pourcentage pas-

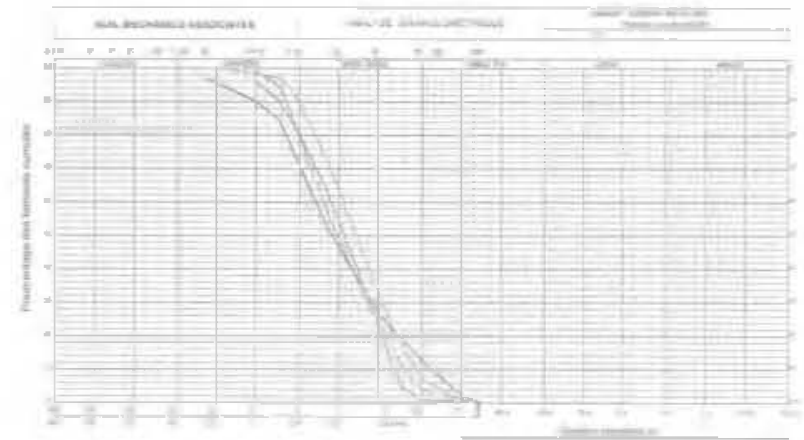

FG ₹ Courbes granulométriques du remblai utilisé. Sieve analysis curves of the used fill.

sant à $80 \mu \mathrm{m}$ étant inférieur à $2 \%$ (Fig. 2). Les différentes courbes granulométriques fournissent un diametre $\mathrm{d}_{\text {5y }}$ compris entre 1 et $2 \mathrm{~mm}$ et un rapport $d_{60} / d$ entre 5 et 10.

\section{2.}

\section{Propriétés mécaniques}

Les propriétés mécaniques du sable ont été caractérisées par des essais de cisaillement à la boîte de Casagrande, en milieu saturé et à une vitesse de $1 \mathrm{~mm} /$ min. Deux séries d'essais ont été exécutées : la première avec des échantillons dans un êtat lâche saturé, obtenu en versant Je matériau directement au nu cle la partie supérieure de la boîte et, la seconde, avec des échantillons dans un état compact saturé en damant le matériau mis dans la boîte au moyen d'un cube en bois au fur et à mesure de la mise en place.
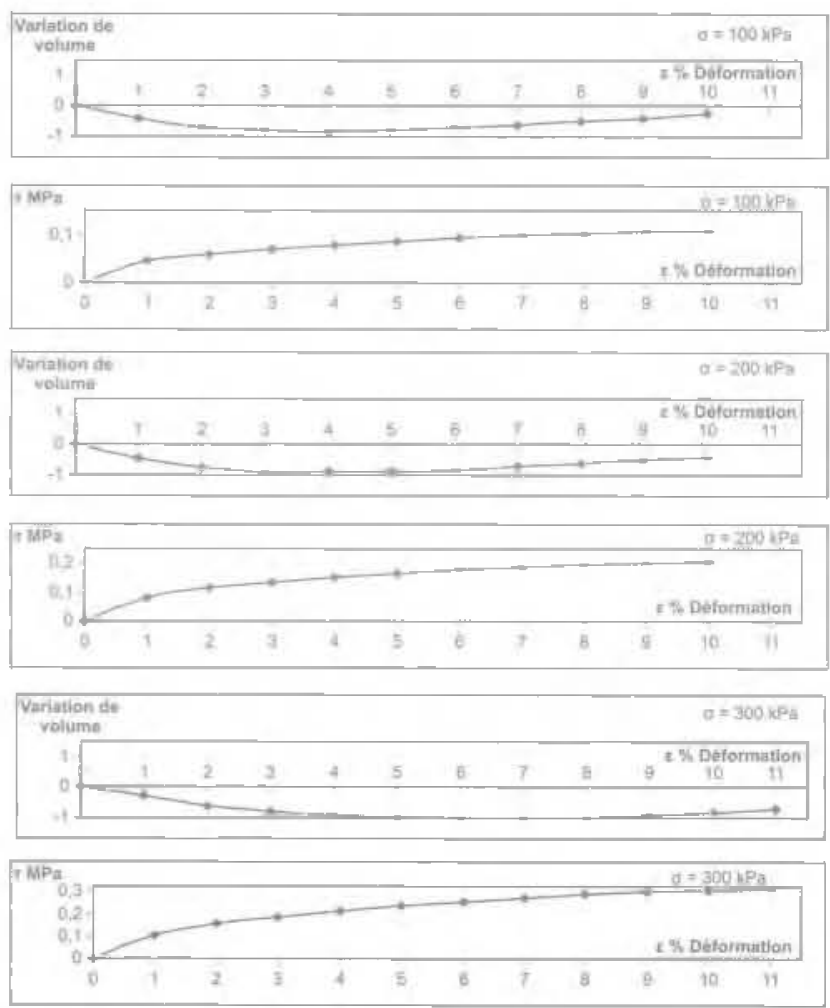

กG. 3 Essais de cisalllement : courbes contraintesdéformations obtenues à l'état lâche saturé. Direct shear tests : stress-strain curves obtained at the saturated loose state. 
Les résultats ohtenus avec nn éfa! lâche du sable n'indiquent pas de rupture franche (Fig. 3). Toutefois, au-dela d'une valeur de 9 à $11 \%$, la déformation augmente de quelques pour cent pour une très faible aug. mentation de la contrainte de cisajllement. Par ailleurs, il se produit une diminution du volume pendant toute la durée du cisaillement : l'angle de frottement interne est voisin de 26 degrés.

Cette valeur de 26 degrés est plausible pour un sable qui vient d'être deversé. D'après les corrélations données dans le document de la Société internationale de mécanique des sols et travaux de fondations intitulé " Utilisation des résultats des essais pressiométriques pour le dimensionnement des fondations en Europe m, elle correspond à une pression limite au pressionètre de $250 \mathrm{kPa}$ (figure 12 du document cité\}, c'est-à-dire à une résistance de pointe ał pénétronètre statique de 1500 à $2000 \mathrm{kPa}$, que l'on a mesurée lors des sondages pénétrométriques.

Pour un état compact du sabie, les courbes contraintes-défomations présentent un pic pour une déformathon comprise entre 6 et $8 \%$, avec une dininution de volume pour les petites déformations et une augmentation ensuite, caractéristique du phénomène de diłatance (Fig. 4). Avec 1'hypothesse d'une cohésion nulle, l'angle de frottement inteme vaut environ 46 degrés, ce qui est relativement important. A cet angle de frottement interne de 46 degrés correspond une pression limite pressionétrique de $2500 \mathrm{kPa}$. c'est-à-dire une résistance de pointe de l'ordre de $20000 \mathrm{kPa}$ qui sera observée lorsque le sable aura été bien compacté par pilonnage.
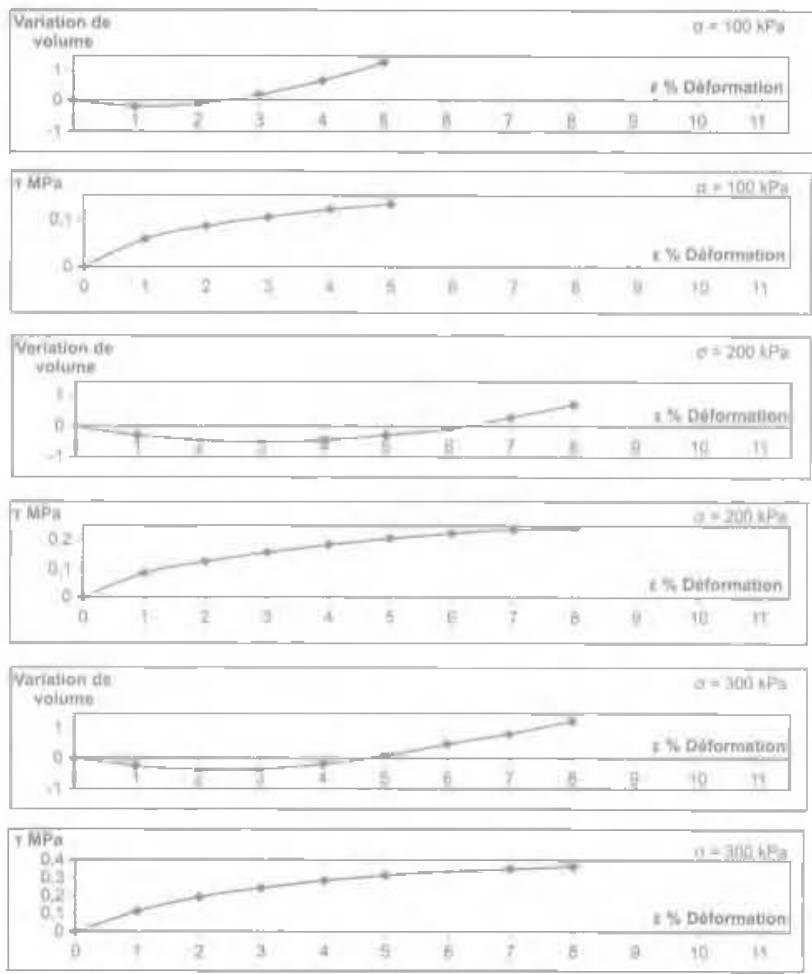

FG. 4 Essais de cisaillement : courbes contraintesdéformationsobtenuesàl'élatcompactsaturế. Direct shear testg : stress-strain curves obtained at the saturated compact state.

Par ailleurs, tout le remblai a été étudié au moyen du pénétromètre statique ANDINA de $150 \mathrm{kN}$, les lectures étant fattes tous les $25 \mathrm{~cm}$ avec une vitesse de pénétration de $2 \mathrm{~cm} / \mathrm{s}$. Les coupes obtenues montrent que l'épaisseur du remblai varie entre 4 m côté Est et $10 \mathrm{~m}$ côté Ouest, le sable êtant légèrement compacté au-dessus du niveau de la mer, avec des résistances de pointe q variant entre 3 et $5 \mathrm{MPa}$, sá résistance en dessous du niveau de la mer variant entre 0,5 et $1,5 \mathrm{MPa}$, ce qui correspond à un sable lậche. En dessous du remblai, on retrouve le sol en place constitué généralement par un sable silteux graveleux dense dont la résistance de pointe varie entre 7 et $20 \mathrm{MPa}$. Deux diagrammes de pénétration type sont présentés sur la figure 5.
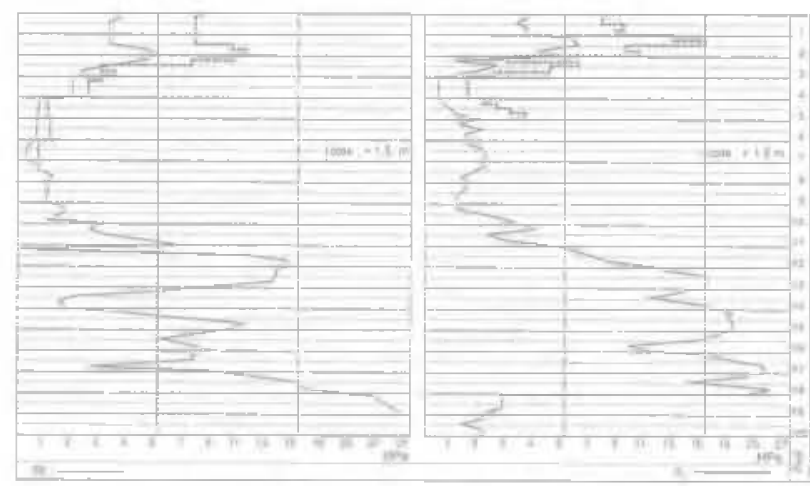

FG 5 Deux coupes type avant traitement. Two typical sections before treatment.

\section{3}

\section{Les risques existants}

a) Avec les caractéristiques mécaniques mesurées dans le remblai, tout chargement statique ne peut qu'entraîner des tassements totaux et différentiels importants. En effet, sì l'on considère un bătiment fondé sur un radier posé sur ce remblaí, le tassement total prévisible sous une contrainte verticale movenne de $150 \mathrm{kPa}$, correspondant grossièrement à un immeuble de 12 étages, peut dépasser le demi-mètre. Ceci est inadmissible et constitue un danger sévère pour la construction érvisagée.

b) Indépendamment de tout chargement, le sable de dragage obéit par ailleurs à tous les critères d"état conduisant à le qualifier de potentiellement liquéfiable :

- degrè de saturation de $100 \%$;

- diamètre $\mathrm{d}_{50}$ voisin de $1 \mathrm{~mm}$ (donc compris entre 0,05 et. $1.5 \mathrm{~mm}$;

- granulométrie uniforme et rappori $\mathrm{d}_{\text {ag }} / \mathrm{d}$ conpris entre 5 et 10 (donc inférieur à 15 ).

Les résistances en pointe obtenues etant celles d'un sable lâche, il est donc à craindre que dans l'état a naturel $\%$ après remblaiement hydraulique, ce sol. soumis a un chargement cyclig̣ue dans un pays classé á sélimicité moyenne, subisse des tassements importants et $\Omega$ atteigne la rupture par liquéfaction.

c) Ies travaux de Seed et al. (1971) ont permis d'estimer le rapport de contrainte cyclique dû à un séisme, $\tau_{a} / \sigma^{\prime}$ oi $\tau_{a w}$ est la contrainte de cisaillement moyenne et $\sigma^{\prime}$ " la contrainte verticale effective à la profondeur considérée. Ils ont proposé une borne inférieure délimitant les cas de non-liquéfaction. Ce rapport $\tau_{\tilde{\sigma} \mathrm{u}} / \sigma^{\prime}{ }_{\mathrm{u}}$ est défini par la relation

$$
\frac{\tau_{\text {av }}}{\sigma_{v}^{\prime}}=0,65 \times \frac{\sigma_{v}}{\sigma_{v}^{\prime}} \times \frac{a_{\max }}{g} \times r_{d}
$$


avec $u_{\text {roax }}$ l'accélération maximale du sol en surface; $\mathrm{g}$, l'accéleration de la pesanteur ; $\sigma_{\gamma}$ la contrainte verticale totale à la profondeur considerée exprimée en $\mathrm{kPa}$ et ${ }^{\mathrm{d}}$, un terme correcteul fonction de la profondeur $z_{\text {, }}$ exprimée en $\mathrm{m}$, de la couche: $r_{d}=1-0,015 \mathrm{z}$.

Quelques années plus tard et, compte tenu de données supplémentaires, Seed et al. (1975. 1983) ont exprimé le rapport $\tau_{7 y} / \sigma^{\prime}$ en fonction d'une valeur corrigée de la valeur SPT et ont proposé de transposer leur critère. développé pour le SPT; au pérétromètre statique. Ils ont ainsi établi une courbe enveloppe délimitant un domaine dans le diagramme $\left(\tau_{z} / \sigma_{v}^{\prime}, c_{1}\right)$, contenant l"ensemble des sites où la liquéfaction a été observée, applicable au sable ou au sable silteux pour des séismes de magnitude 7,5 . Cette courbe est approchée par l'équation (2):

$$
\left(\frac{\tau_{\mathrm{gy}}}{\sigma_{\mathrm{v}}^{\prime}}\right)_{1}=\frac{\mathrm{q}_{\mathrm{c}}}{45}
$$

où $\mathrm{q}_{\mathrm{r}}$ est exprimée en $\mathrm{MPa}$.

En dessous de cette courbe, il n'y a pas de liquéfaction alors qu"au-dessus il y a liquéfaction. Par ailleurs, des coefficients correcteurs ont été introduits par Gorté (1982) pour exprimer l'influence de la magnitude sur la résistance à la liquéfaction.

Pour appliquer la démarche proposée par Seed et al, il convient d'estimer le paramètre a. Comme il nexiste pas au Libar de carte sismotectonique figurant les intensités maximales ressenties, Jes épicentres, etc., on a procédé par analogie. On sait ainsi que le Liban a été soumis à des secousses sismiques. La derniêre en date ả avoil provoqué des destructions dans les constructions rurales (type A) est celle de 1956. Son intensité était de VI dans l'échelle MSK et sa magnitude de 5,6 dans l'échelle de Richter:

Les bâtiments prévus dans la Marina étant en béton armé bien structuré (type C), il faut donc que l'intensité de la secousse soit au moins de VI dans l'échelle MSK pour que ce type de bâtiment subisse des dommages légers, du premier degré. C'est à partir de cette valeur d’intensité que la vulnérabilité du bâtiment peut être dêduite par l'application des règles de construction parasimique. Apres discussion avec l'entreprise Khoury, il a été décidé d'évaluer le risque de liquéfaction du remblaj pour une intensité de VII dans l'échelle MSK. A cette intensité, il taut faire correspondre une valeur de l'accélération maximale a qui, d'après Willmore (cité par. Davidovici, 1985) serait comprise entre 100 et $200 \mathrm{~cm} / \mathrm{s}^{2}$, soit un rapport $\mathrm{a}_{\mathrm{max}} / \mathrm{g}$ variant entre 0,1 et 0,2

Pour l'Europe du Sud, la corrélation entre l'intensité VIIl dans l'échelle MSK et laccélération a implique une dersité de probabilité de $a_{\text {max }}$ ëgale à 1 pour $a_{\text {mar }} / g=0,30$. d'après Davidovici (1985)

Dans l'impossibilité de disposer de données locales, on a choisi avec l'approbation du Promoteur de mener les calculs pour a $/ g=0,2$ et a $/ g=0,1$. Dans ces conditions, le calcul montre que, dans la zone d'épaisseur des sables lâches de dragage, tous les nivezux sont pratiquement liquéfables, que le rapport $\mathrm{a}_{\mathrm{max}} / \mathrm{g}$ soit égal à 0,2 ou a 0,1 .

\section{4}

\section{Choix de la méthode d'amélioration pour les remblais}

Deux méthodes d'amélionation ont été utilișées le vibrocompactage et le pilonnage intensif.

\section{Vibrocompactage}

Les opérations de vibrocompactage ont été réalísées avec un vibroflotteur PENNINE Hydraulique type 130HD dont les caractéristiques sont les swivantes

$\begin{array}{ll}\text { Force centrifuge } & 140 \mathrm{kN} \text { à } 3000 \text { RPM } \\ \text { Fréquence } & 50 \mathrm{~Hz} \\ \text { Amplitucle } & 13 \mathrm{~mm} \\ \text { Diamètre } & 292 \mathrm{~mm} \\ \text { Masse } & 28 \mathrm{kN} \\ \text { Puissance du moteur } & 100 \mathrm{~kW}\end{array}$

La techrique de vibrocompactage retenue est la suivante.

Le vibroflotteur, tenu par une grue, penètre par vibration et traverse la partie lâche du sol. Une fois gutil atteint la profondeur requise, nomalement le sable compact en place, il commence à être retíré et un tout venant grossier est introduit pour aider au compactage du sable läche, et remplacer le volume perdu par densification.

Lopération se poursuit jusqu'à ce que la colonne de tout venant ainsi formée atteigne le niveau du terrain en place. L'operation est répétée suivant une maille à déterminer pour que les effefs de la vibration, conjugués à l'introduction d'un matériau hautement frottant. donnent le meilleur rendement allié à une efficacité suffisante.

A cet effet, des plots d'essai prélininaires ont até réelisés par l'entreprise pour fixer cette maile et pour mesurer. suivant la rapidité de descente ou d'extraction de la sonce, l'amélioration obtenue. Ces essais ont conduit à faire varier la maille de compactage de $1,7 \mathrm{~m} \times 1,7 \mathrm{~m}$ à $1,5 \mathrm{~m} \times 1 \mathrm{~m}$ ainsi que le délai de repos, après la réalisation de ce compactage, entre deux et quinze jours. Les essais ont été exécutés dars la parcelle 768 (Fig. 6) et ont donné :

- pour la maille de $1,7 \mathrm{~m} \times 1.7 \mathrm{~m}$, dans la couche de remblai de surface, une résistance de pointe moyenne de $6 \mathrm{MPa}$ (Fig. 7 ) :

- pour la maille de $1.5 \mathrm{~m} \times 1.5 \mathrm{~m}$, des résultats généralement meilleurs avec une résistance de pointe moyenne en surface égale à $8 \mathrm{MPa}$ ou plus, mals certains sondages sont moins concluants ;

- pour la maile de $1.5 \mathrm{~m} \times 1 \mathrm{~m}$. la résistance en pointe movenne est supérieure d. $9 \mathrm{MPa}$ (Fig. 8).

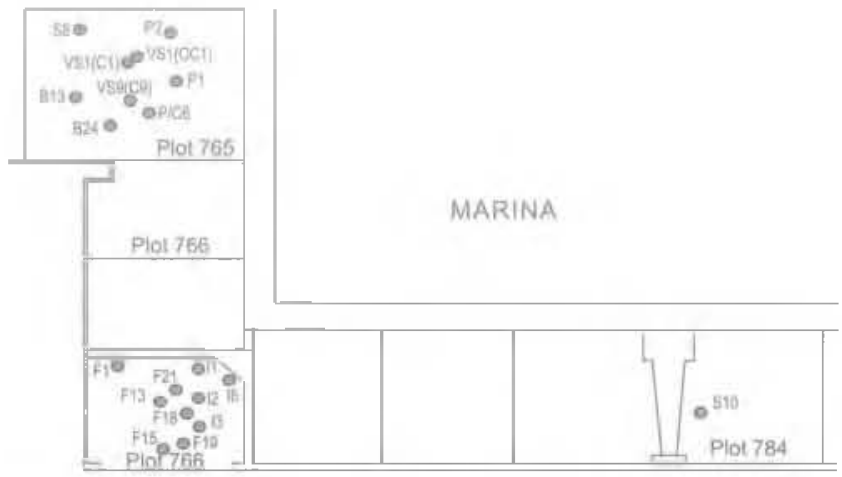

FIG. \& Positions des parcelles étudièes par rapport ả lamarinaetimplantationdessondagesd'essai. Position of the studied plots with respect to the marina and set up of boreholes. 

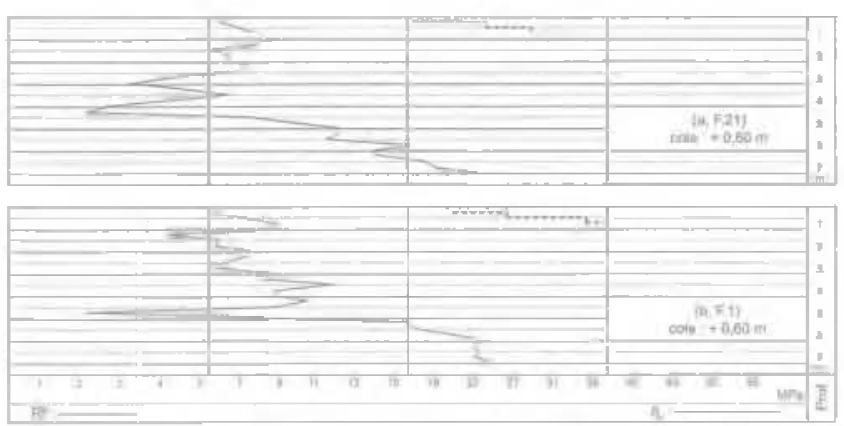

flG. 7 Vibrocompactage : amélioration obtenue pour une mailie de $1,7 \mathrm{~m} \times 1,7 \mathrm{~m}$ (a, F,21) et une maille de $1,5 \times 1,5 \mathrm{~m}(\mathrm{~b}, \mathrm{~F}, 1)$.

Vibro-compaction : improvement obtained for a grid of $1.7 \mathrm{~m} \times 1.7 \mathrm{~m}(\mathrm{a}, \mathrm{F} .21)$ and a grid of 1.5 m7 $\times 1.5 \mathrm{~m}(\mathrm{~b}, \mathrm{~F} .1)$

Il convient de noter gue, d'aprẻs l'entreprise Khoury, des dépots de limon, inhérents à la technique du remblaiement hydraulique, auraient été frequents à l'emplacement de cette parcelle, ce qui explique l'amélioration un peu rnoins marquée des caractérístiques du remblai.

Par ailleurs, certains sondages, réalisés près de la colonne de gravier et y pénétrant plus ou moins, ont donné des résistances de pointe très fortes, quí de ce fait ne sont pas représentatives de l'ensemble du terrain.

Sur la base de ces essais, i] a été décidé de poursuívre le vibrocompactage sur le restant de la parcelle en adoptant une maille de $1.7 \mathrm{~m} \times 1.5 \mathrm{~m}$.

Deux semaines après la fin de l'opération, six sondages pénétrométriques de contrôle (série I) ont étê

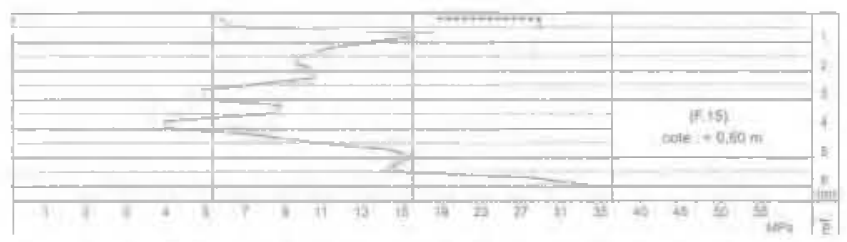

คธ. Vibrocompactage : amélioration obtenLe pour une maille de $1,5 \mathrm{~m} \times 1,0 \mathrm{~m}$ (F.15)

Vibro-compaction : improvement obtained fot a grid of $1.5 \mathrm{~m} \times 1.0 \mathrm{~m}(\mathrm{~F} .15)$.

exécutés à la cote $+0,6$ environ, c'est-à-dire après obtention dur tassement apparent de $70 \mathrm{~cm}$.

A l'exception d'un sondage qui montre à partir de $2 m$ de profondeur la présence de limon sur $3 m$ d'épaisseur, les résistances moyennes en surface sont supérieures à $8 \mathrm{MPa}$, une couche d'argile silteuse, de 1 m d'épaisseur et de résistance de pointe q égale à $3 \mathrm{MPa}$, etant parfois notee [Flg. 9),

On en a donc conclu que, par vibrocompactage et pour des épaisseurs de remblai de l'ordre de $8 \mathrm{~m}$, le tassement maximal que l'on pouvait obtenir en surface avec le matériel choisi était compris entre 60 et $70 \mathrm{~cm}$.

\section{2}

\section{Compactage par pilonnage intensif}

Le compactage par pilonnage intensif a été peu utilisé au Liban, du fait essentiellement de l'absence de projets importants nécessitant son utilisation pendant la guerre entre 1975 et 1990. Juste avant le début de cette période, en 1974, nous avions étudiè avec Louis
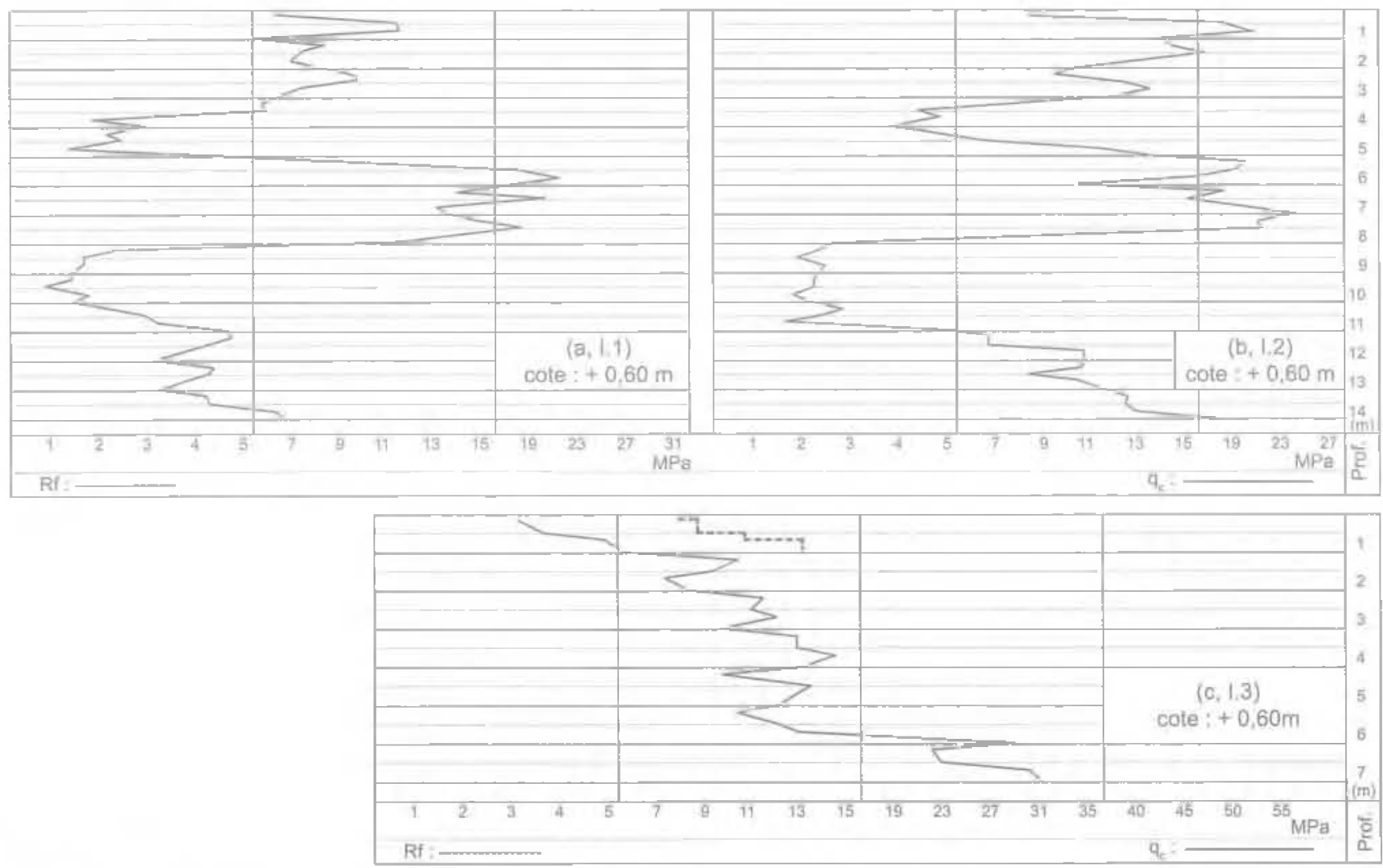

Flc. 5 Vibrocompactage : amélioration obtenue pour une maille de $1,7 \mathrm{~m} \times 1,5 \mathrm{~m}$ (a, I.1).

Vibro-compaction : improvement obtained for a grid of $1.7 \mathrm{~m} \times 1.5 \mathrm{~m}(\mathrm{~s}, 1.1 \mathrm{f}$. 
Ménard la nossibilité d'utiliser cette technirup à l’usine de traitement des ordures ménagères à la Quarantaine dans Beyrouth. Mais le déclenchernent des opérations militaires et la disparition brutale de Louis Mérard mirent fin à ce projet.

Plus tard, au cours de la guerre, en 1981, sur le site du centre balnéajre de Rimal, nous avons fabriqué un pilon de $80 \mathrm{kN}$ chutant de $6 \mathrm{~m}$ à l'aide de grues de fortune. Le but etait de compacter un remblal de 4 a $5 \mathrm{~m}$ d"épaisseur déversé sur une surface de $6000 \mathrm{~m}^{2}$ sur lequel devaient reposer la piscine olympique et les cabines. Ce traitement avait conduit multiplier par 3 au moins le module initial moyennant un tassement en Surface de 30 à $40 \mathrm{~cm}$ représentant une réduction de volume du remblai de l'ordre de $8 \%$. Il permit de réaliser des fondations superficielles chargées, en semice, a $300 \mathrm{kPa}$.

Cette techmique fut reprise lors de la construction d'un remblai entre Antelias et Dbayé. Les premières opérations de pilonnage furent ainsi réalisées avec le même pilon de $80 \mathrm{kN}$, ayant une aire d'impact de $2,25 \mathrm{~m}^{2}$ et tombant une dizaine de fois de $8 \mathrm{~m}$ environ, en appliquant une énergie de $220 \mathrm{tm} / \mathrm{m}^{2}$. Le tassement obtenu avec cełte érnergie était voisin de $60 \mathrm{~cm}$.

Le résultat fourni par un essai pénétrométrique, réalisé près de $\mathrm{S} .1$ (noté contrôle V/S.1(C.1). figure 10). a montrế une anélioration certaine sum les trois mètres supérieurs mais la résistance ce polnte ne s'éloignait pas beaucoup èn profondeur de celle mesurée avant le compactage.

Il fut donc décidé d'utiliser un nouveau piton. confec tionné par l'Entreprise Khoury, ayant une masse de $220 \mathrm{kN}$ et une aire d'impact de $4 \mathrm{~m}^{2}$. Cette masse a été lancée une dizaine de fois de $10 \mathrm{~m}$ de hauteur jusqu'à ce rute ann enfrncement atteigne une cote voicine de $+0,3$ et des essais de contrôle ont été exécutés [Fig. 10]. Ils sont repérés par les notations V/S1 (CC1) et V/S9 (C9). Ces essais ont montré que l'anélioration touchait maintenant 4 à $4,5 \mathrm{~m}$ en surface, mais qu'il restait entre 1 et $1,5 \mathrm{~m}$ de sable silteux plus ou moins làche.

I'énergle de compactage a ensuite été augmentée pour atteindre la cote $-0,3$ et a ce stade un sondage réalisé en $\mathrm{P} / \mathrm{C} .6$ a indiqué que le compactage avait fait passer la résistance de ce dernier mètre de sable silteux lâche à une valeur moyenne de $3 \mathrm{MPa}$, jugêe acceptable (Fig. 11).

Le résultat ayant été concluant, il fut donc décidé d'atteindre la cote $-0,3$ pour le remblai sableux.

Dans le même temps, on a mesuré la résistance đu remblai en tout venant de carrière, au moyen du pénétrometre statique et par carottage avec essai pressiométrique Ménard. Du fait de la présence de blocs, trois des pénétrations réalisées à partir de la plateforme $+1,3$ ont rencontré un refus à 2 m de profondeur, alors que le sondage par carottage exécuté a partir de la même coté montrait a ce niveau ces pressions limites élevées, reflètant probablement la présence de ces bioss. Par allleurs, au-delà de $4 \mathrm{~m}$ de profondeur, il a été impossible de réaliser le moindre essaj à cause des éboulements quí se produisaient. Ces essais pressiométriques n'apportèrent donc pratiquement aucun résultat.

En revanche, les sondages pénétrométriques exécutés à partir de la cote + 0.3, c'est-à-dire après conpactage ( $\mathrm{P} 1$ et $\mathrm{P} 2$ ), ont montré que le remblai avat des caractéristiques moyennes à bonnes (Fig. 12), et que, de ce fait, une poursuite éventuelle du compactage jusqu’a la cote - 0,3 ne pouvait cu'anéliorer encore les résultats obtenus.

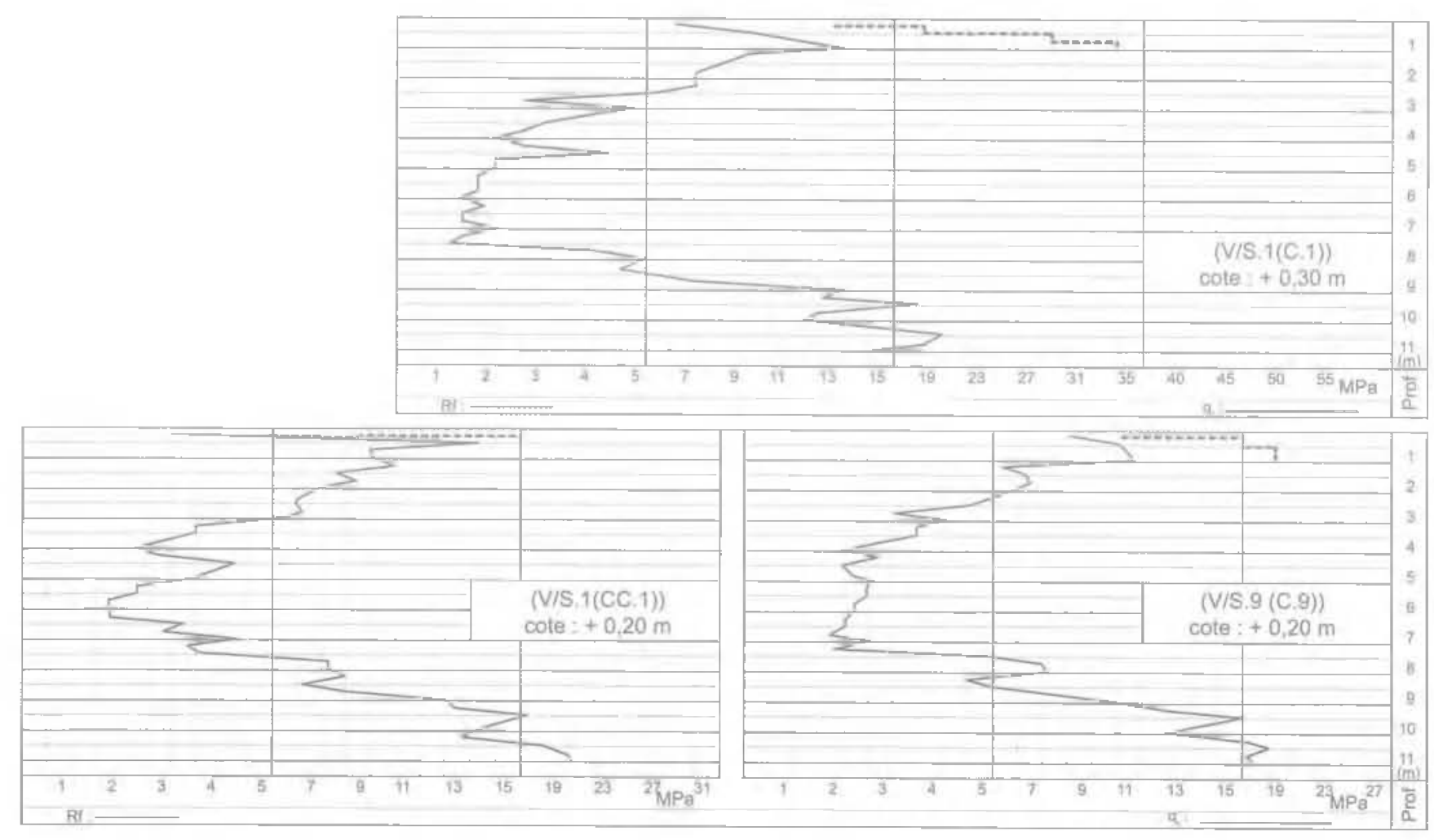

FIG. 10 Pilonnage intensif : amélioration obtenue avec un pilon de $80 \mathrm{kN}$ chutant de $8 \mathrm{~m}$ (V/X(C.1)) et avec un pilon de $220 \mathrm{kN}$ chutant de $10 \mathrm{~m}$ pour atteindre la cote $+0.3(\mathrm{~V} / \mathrm{S} .1[\mathrm{CC} .1)]$ et $(\mathrm{V} / \mathrm{S} .1[\mathrm{C} .9)\}$.

Dynamic compaction : improvement obtained with an $80 \mathrm{kN}$ earth rammer falling from 8 m lreight (V/S. 1(C. 1)) and with a $220 \mathrm{kN}$ earth rammer falling from $10 \mathrm{~m}$ to reach level +0.3 (V/S. 1(CC.1) and IV/S.1(C.9)). 


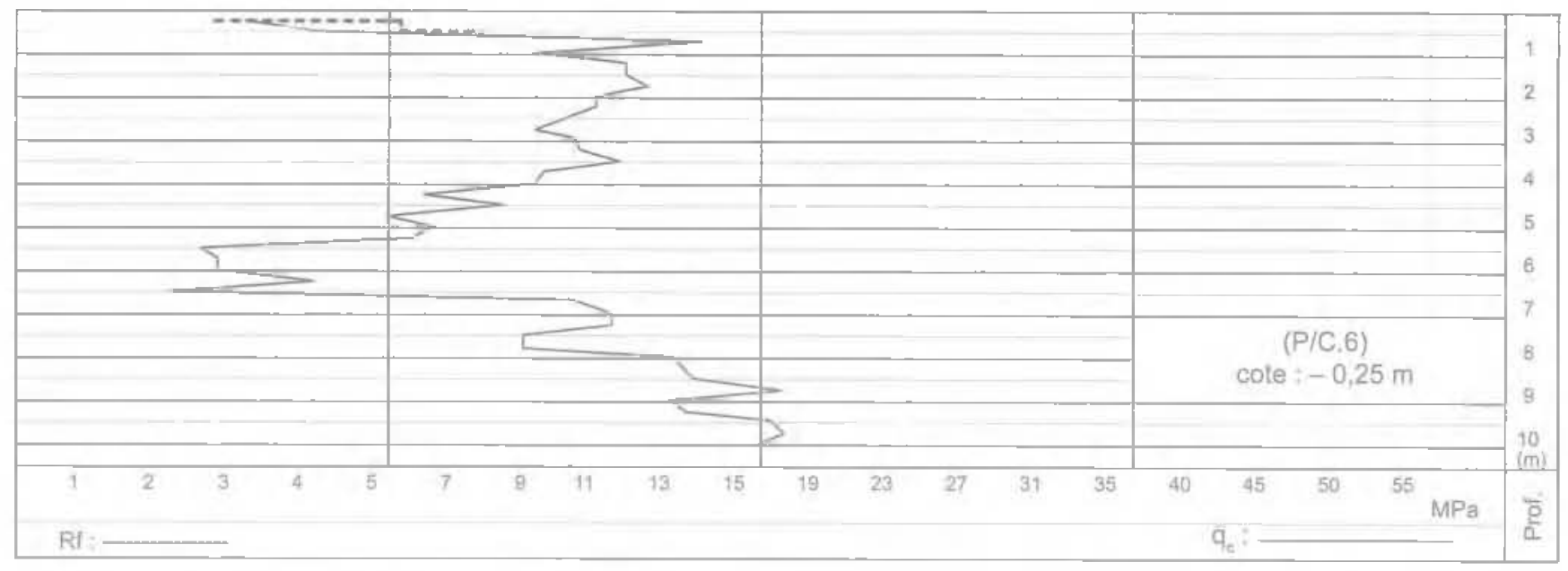

FIG. 11 Pilonnage intensif : amélioration avec ur pilon de $220 \mathrm{kN}$ chutant de $10 \mathrm{~m}$ pour atteindre la cote $\rightarrow 0,3[\mathrm{P} / \mathrm{C} .6]$. Dynamic conpsction : improvenent with a 220 kN earth ramner failing fron $10 \mathrm{~m}$ to reach level -0.3 (P/C.6).
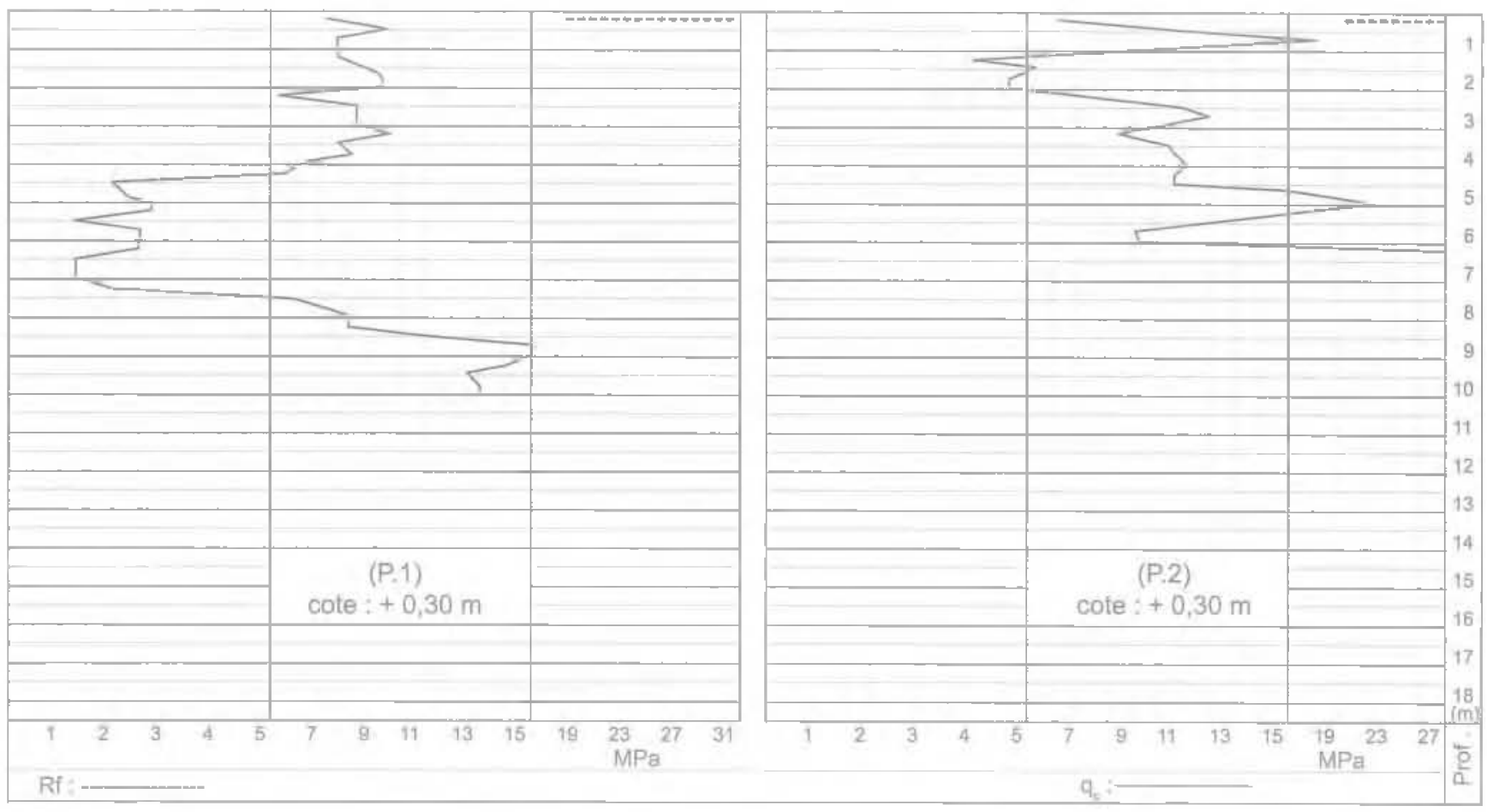

FIG. 12 PiJonnage jntensif : amélioration dans le remblai de carrière (P.1) et [P.2).

Dynamic compaction : improvement in the quarries" fïl (P.1) and (P.2).

Dans ces conditions, l'entreprise a décidé d'étendre à toute la parcelle concernée l'objectî́ à atteindre, c'est à dire la cote $-0,3$ pour les zones sableuses et $+0,3$ ou en dessous pour les zones en tout venant. On est ainsi parvenu à réduire de $1,6 \mathrm{~m}$ l'épaisseur du remblai. permettant à la résistance de pointe d'atteindre 12 à $15 \mathrm{MPa}$ en surface et ả réduire à $1 \mathrm{~m}$ au plus l'épaisseur du limon rencontré tout en augmentant sa résistance de pointe jusquàa $4 \mathrm{MPa}$ (Fig. 13).

\section{3}

\section{Mode de vérification dans les enrochements}

Si, dans le sable, le pénétromètre statique dynamique ANDINA a pu être utilisé, cet appareil s'est révélế inefficace quand il s"est agi de tester les enrochements.
Pour vérifier l'état de compactage de ces enrochements, on a estimé que l'enregistrement des paramètres de forage (notamment la vitesse d'avancement à pression constante sur l'outili) pouvait indiquer, au moins qualitativement, le degré de compactage. Pour cẻa, on a commencé par réaliser des diagraphies Lutz dans un remblai rocheux, à l'air, provenant de la même carrière. Les vitesses notées varjent entre 150 et $250 \mathrm{~m} / \mathrm{h}$, celles obtenues en traversant un bloc étant comprises entre 10 et $20 \mathrm{~m} / \mathrm{h}$.

Au fur et à nesure que les élêments de rocher deviennent serrés, en fonction du degré de compactage, les vitesses précédentes décroissent et on constate, apres dix chutes du pilon de $150 \mathrm{kN}$, des valeurs évoluant entre 50 et $100 \mathrm{~m} / \mathrm{h}$.

Par tranches de $30 \mathrm{~cm}$ de hauteur, on a évalué la fréquence des valeurs de vitesse de pénétration, notées jusqu'à $12 \mathrm{~m}$ de profondeur. Cette étude montre que : 

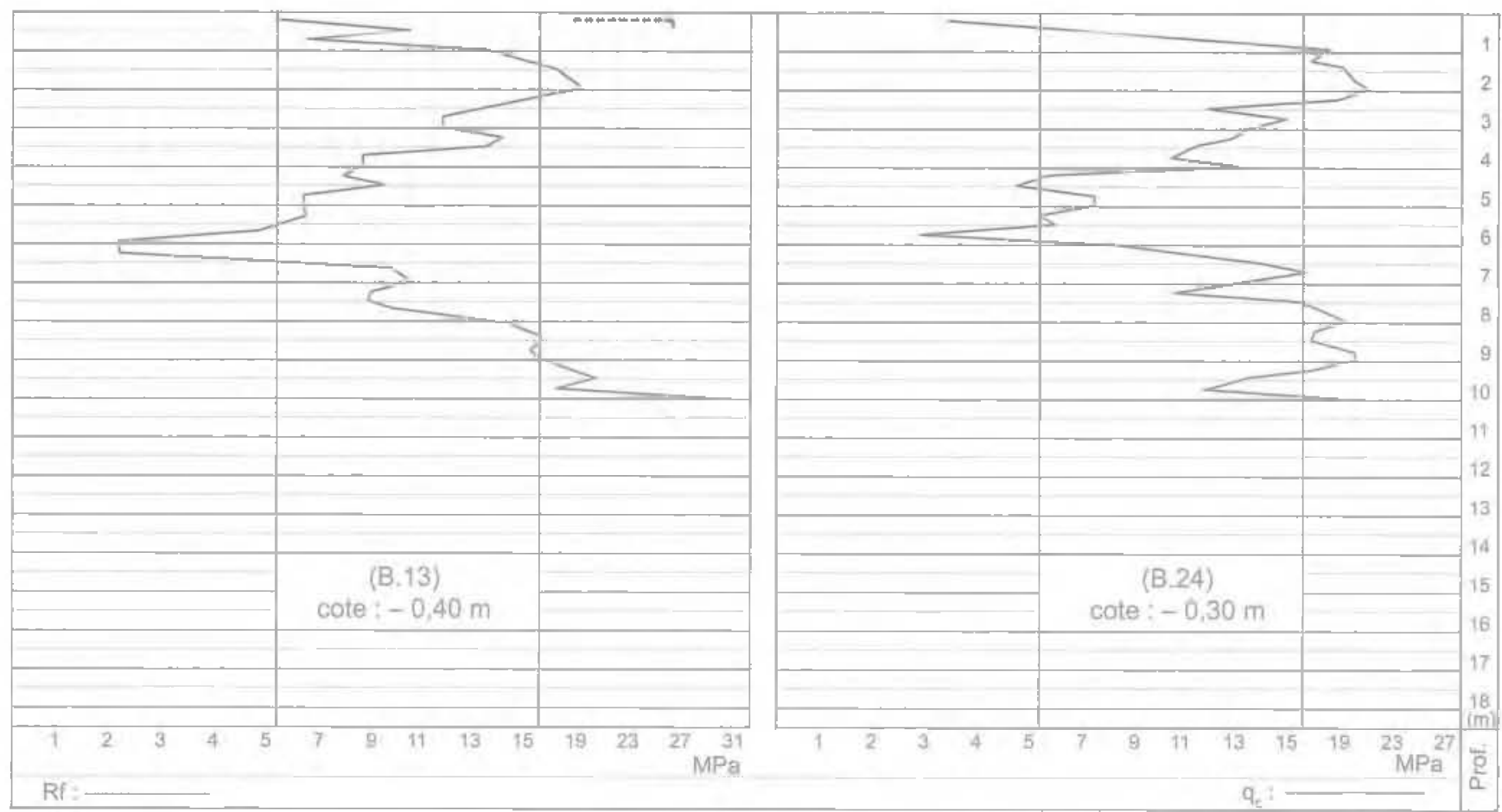

FG. 13 Vibrocompactage et pilonnage intensif : amélioration obtenue après un tassement de 1,6 m) (B.13) (ct (B.24). Vibro-compaction and dynamic compaction : improvement obtained after reaching $1.6 \mathrm{~m}$ of settlement (B. 13 ) and (B.14).

- les valeurs comprises entre 0 et $50 \mathrm{~m} / \mathrm{h}$ représentent entre 7 et $20 \%$ des valeurs obtenues;

- celies comprises entre 50 et $100 \mathrm{~m} / \mathrm{h}$ constituent 30 à $60 \%$ des valeurs, cette demière valeur étant notée proche de la surface et en profondeur :

- celles comprises entre 100 at $150 \mathrm{~m} / \mathrm{h}$ représentent 20 a $40 \%$ des valeurs:

- celles comprises entre 150 et $200 \mathrm{~m} / \mathrm{h}$ représentent 15 道 $30 \%$ des valeurs;

- celles comprises entre 200 et $250 \mathrm{~m} / \mathrm{h}$ argile ou sable) ne dépassent pas $5 \%$.

\section{5}

\section{Validation des calculs avec des mesures de tassement en vaie grandeur}

\section{1}

\section{Estimation des modules de déformation Ménard}

Les modules de déformation. Ménard ont été esti mês. à partir des résultats pénétrométriques statique ANDINA, de la maniêre suivante :

- pour les sables, la pression limite a été calculée à l'aide de la relation $\mathrm{p}_{1}=\mathrm{q} / 12$ (CTRE, 1991) et on a considéré, pour les couches de surface, cue le module de déformation $\mathrm{E}_{\mathrm{M}}$ est égal à $15 \mathrm{p}$, le coefficient de structure étamt priśégal à 0,5 (CEGP, 1968) ;

- pour la couche de limon, la pression limite est égale à $\mathrm{q} / 6$ (CTRE, 1991), et le module $\mathrm{E}_{\mathrm{M}}$ à $12 \mathrm{p}_{\mathrm{I}}$, le coeffictent de structure êtant égal à 0,5 (CEGP, 1968) :
- au-delâ de -16,5 m de profondeur. le module de déformation Ménard est pris égal à $50 \mathrm{MPa}$

Le tassement total est ensuite calculé par la relation :

$$
\omega=\frac{2 q B_{0}}{q E_{d}}\left(\lambda_{d} \frac{B}{B_{0}}\right)^{\alpha}+\frac{\lambda_{c} q B}{q E_{c}} \alpha
$$

$\mathrm{E}_{\mathrm{c}}$ est le module Ménard de la zone où domine la composante isotrope des contraintes et correspond à la moyenne harmonique des nodules sur une épaisseur de sol égale à la demi-largeur clu radier. E est le module Ménard correspondant à la zone oủ domine la composante déviatorique dés contraintes ; son expressian est donnée par:

$$
\frac{1}{E_{d}}=\frac{1}{4}\left\{\frac{1}{E_{c}}+\frac{1}{0,85 E_{2}}+\frac{1}{E_{3 / 4 / 5}}+\frac{1}{2,5 E_{6 / 7 / 8}}+\frac{1}{2,55_{9 / 16}}\right\}
$$

$\mathrm{E}_{\text {piq }}$ est la moyenne harmonique des modules dans les couches pà q.

\section{2}

\section{Étude de la parcelle 765}

Sur la parcelle 765 , traitée en premier par pilonnage intensif avec un tassement du remblai de $1,5 \mathrm{~m}$. quatorze sondages pénétronétriques ont été exécutés: hult en septembre 1998 et six autres en janvier 1999 , c'est-à-dire sept à onze mois après le traitement. Ils confirmèrent, d’une part. l'amélioration déjà apportée lors clu pilonnage et, d'autre part, montrèrent que, par 


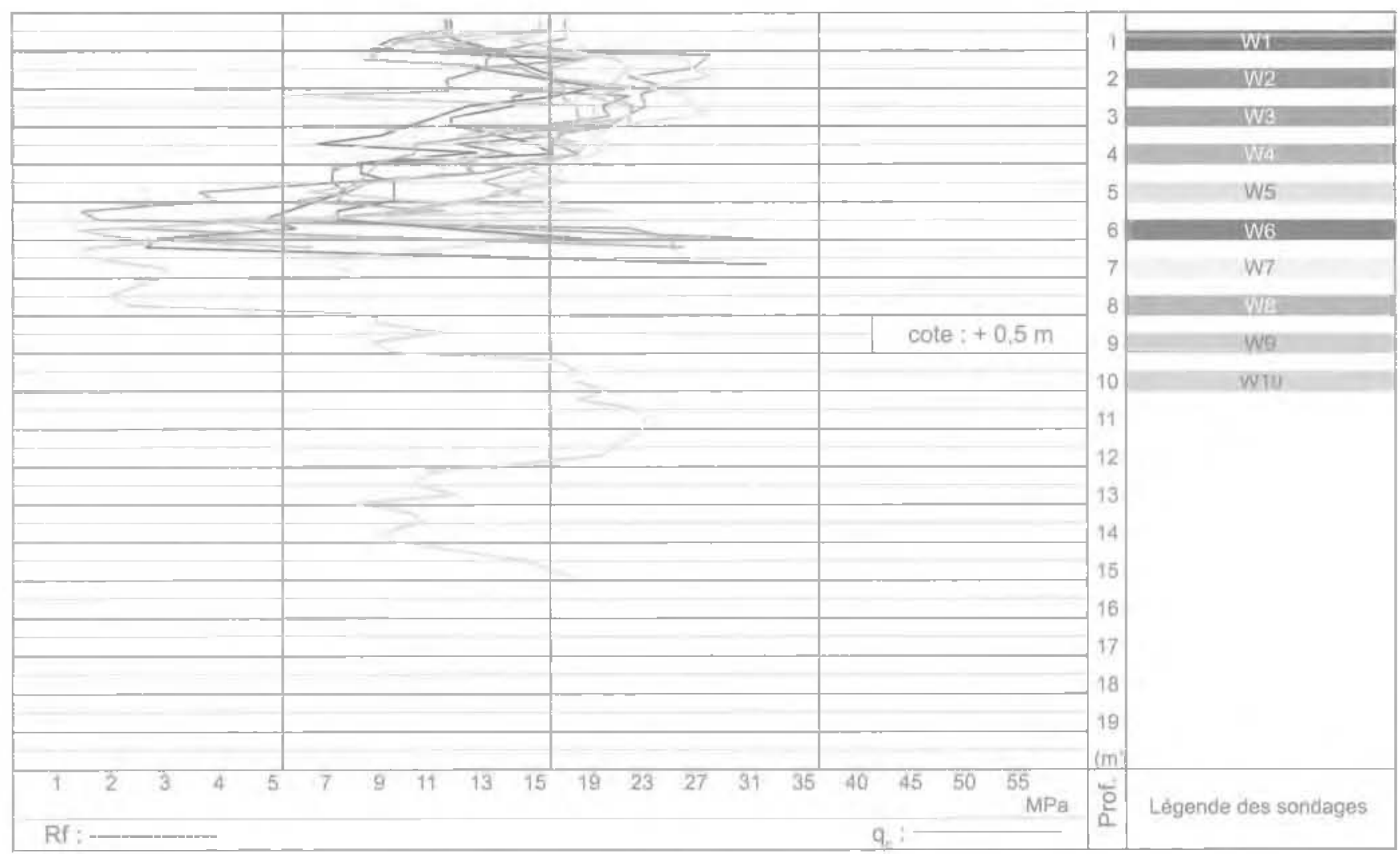

FGG 14 Résultats regroupẻs de 10 sondages effectués sur la parcelle 765, vingrt-cinq mois après le traitement [W.1 à W.14).

Joined results of 10 soundings realized on plot 765. twenty-five montbs after treatment [W.1 to W.14)

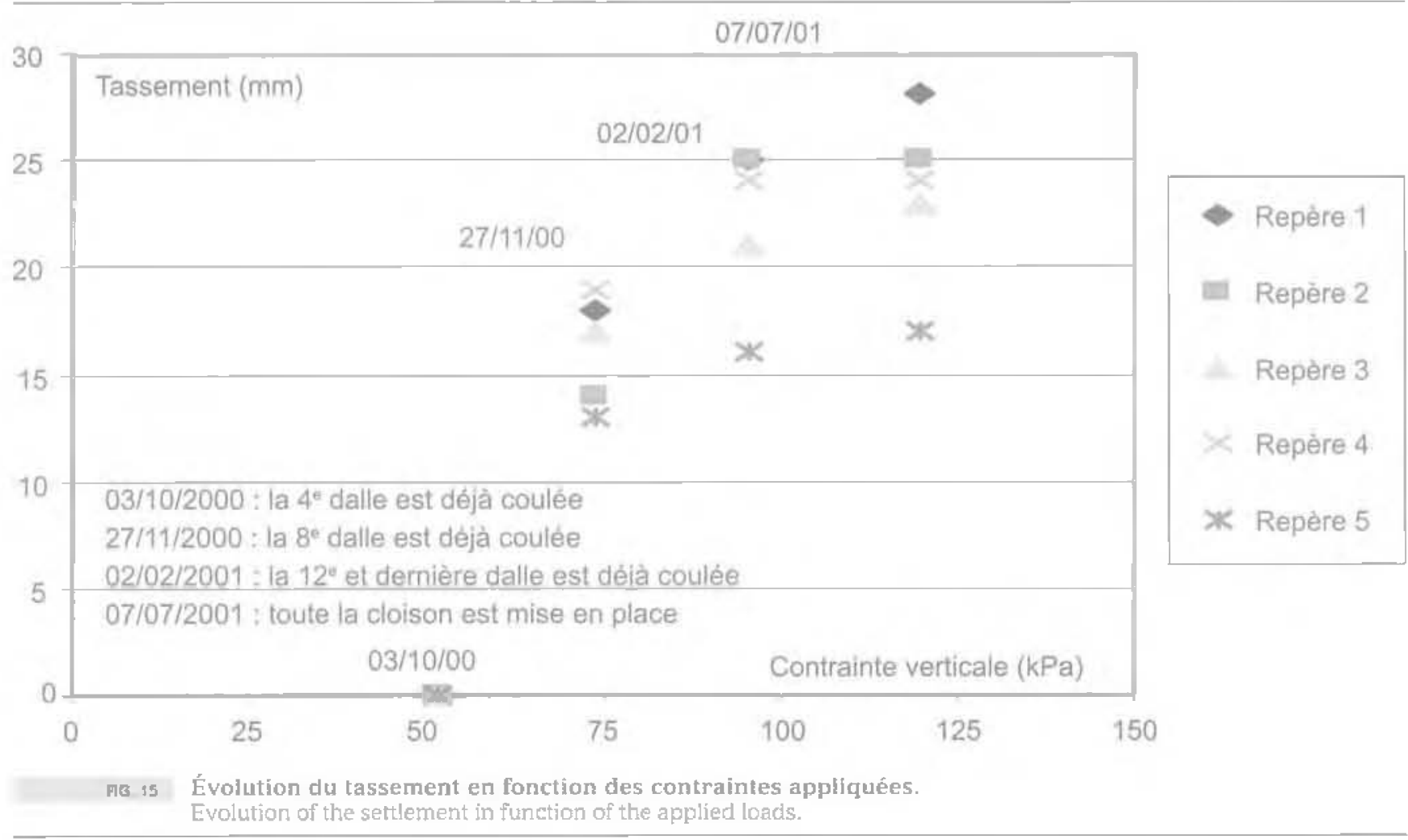

rapport aux résultats déjă obtenus, la résistance avait augmenté avec le temps, mais sans que J'on ait trouvé de loi précise pour cette amílioration.

S'agissant de constmire un inmeuble d'habitation sur une surface de $30 \mathrm{~m} \times 65 \mathrm{~m}$ reposant sur un radier de $1,2 \mathrm{~m}$ d'épaisseur, posé à la cote $+0,5$, avec deux sous-sols, un rez-de-chaussée et neuf étages, dix autres sondages au pénétromètre ANDINA ont été effectués, au droit de l'immeuble, juste avant le dêbut des travaux, c'est-à-dire vingt-cing mois après le traitement. Les résultats ont été regroupés sur la figure 14. Par ailleurs, les calculs de tassement basés 
sur les modules de déformation Ménard montrèrent que l'on devait s'attendre à un tassement total moyen de 3 à $4 \mathrm{~cm}$ pour la coupe de sol décrite dans le tableau I. Les modules de déformation Ménard ont été obtenus par corrélation. TABLEAUI Modules de déformation Ménard $\mathrm{E}_{\mathrm{M}}$
Parcelle 765 .

Ménard deformation moduli $\mathrm{E}_{\mathrm{M}}$. Plot 765 .

\begin{tabular}{|c|c|c|c|c|c|c|}
\hline $\begin{array}{l}\text { ['rofondeul' } \\
\text { (m) }\end{array}$ & $\begin{array}{c}+0,5 \\
a t-1.5\end{array}$ & $\begin{array}{l}-1,5 \\
\text { et }-3,5\end{array}$ & $\begin{array}{l}-3,5 \\
\text { et }-4.5\end{array}$ & $\begin{array}{l}-4,5 \\
\text { et }-11,5 \mathrm{e}\end{array}$ & $\begin{array}{l}-11,5 \\
\text { et }-15,5\end{array}$ & $\begin{array}{l}-15,5 \\
\text { etoura } \\
\text { delä }\end{array}$ \\
\hline $\mathrm{E}_{\mathrm{M}}[\mathrm{MPa}]$ & 20 & 30 & 10 & 30 & 15 & $>50$ \\
\hline
\end{tabular}

Cing repères avaient été installés au pied de l'immeuble pour suive le tassement avec la montée en chargè. En réalité, les premières mesures n'ont vaiment commencé è être prises quaprès le coulage de la quatrième dalle.

Lévolution du lassement en fonction des contraintes appliquées est donnée sur la figure 15 oủ l'on a regroupé l'ensemble des mesures affectuées sur les cinq repères. Elle montre qu'à lá fin de la construction de la structure, le tassement mesuré a varié, suivant les repères, entre 20 et $30 \mathrm{~mm}$.

Après les finitions, quelques millimètres de tassement supplementaires sont apparus confirmant le bien fondé des hypothèses faites sur les valeurs des modules retenus.

\section{Étude de la parcelle 766}

Sur la parcelle voisine 766, OTCE Liban a êtudié un bâtiment de 12 étages construjt sur un radier de $1,3 \mathrm{~m}$ d'épaisseur. Pour les calculs, ce radier a été assimilé ane plaque souple sur ressorts avec un module de réaction du sol de $6 \mathrm{MN} / \mathrm{m}^{3}$.

Là aussi, quinze sondages pénétrométriques ont été réalisés en 2002,46 mois après la fin du traitement. Les résultats sont regroupés sur la figure 16 . Les modules de deformation Ménard nécessaires au calcul du tassement sont indiqués dans le tableau II.

TABLEAU II Modules de déformation Ménard $\mathrm{E}_{\mathrm{M}}$. Parceille 766.

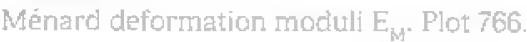

\begin{tabular}{|c|c|c|c|c|c|c|c|}
\hline Profondeur & $\begin{array}{l}+0,5 \\
\text { et } \\
-2,5\end{array}$ & $\begin{array}{l}-2,5 \\
e t \\
-4,5\end{array}$ & $\begin{array}{c}-4,5 \\
\text { et } \\
-5,5\end{array}$ & $\begin{array}{c}-5.5 \\
\text { et } \\
-9.5\end{array}$ & $\begin{array}{c}-9,5 \\
-11,5 \\
-11,5\end{array}$ & $\begin{array}{c}-11,5 \\
\text { et } \\
-16,5\end{array}$ & $\begin{array}{c}-16,5 \\
\text { et au- } \\
\text { dera }\end{array}$ \\
\hline & 15 & 11 & 8 & 20 & 10 & 20 & $>50$ \\
\hline
\end{tabular}

D’après l'étude de OTCE, la contrainte maximale sous le radier aux états limites de service ne dépasse pas $0,25 \mathrm{MPa}$, ce qui est acceptable devant une capacité portante de service de 0,3 $\mathrm{MPa}$, le tassement total prévisible étant de 35 min.

Deux mesures faites au début et à la fin de la construction ont indiqué un léger tassement, de l'ordre de quelques millimètres. Une contre-expertise

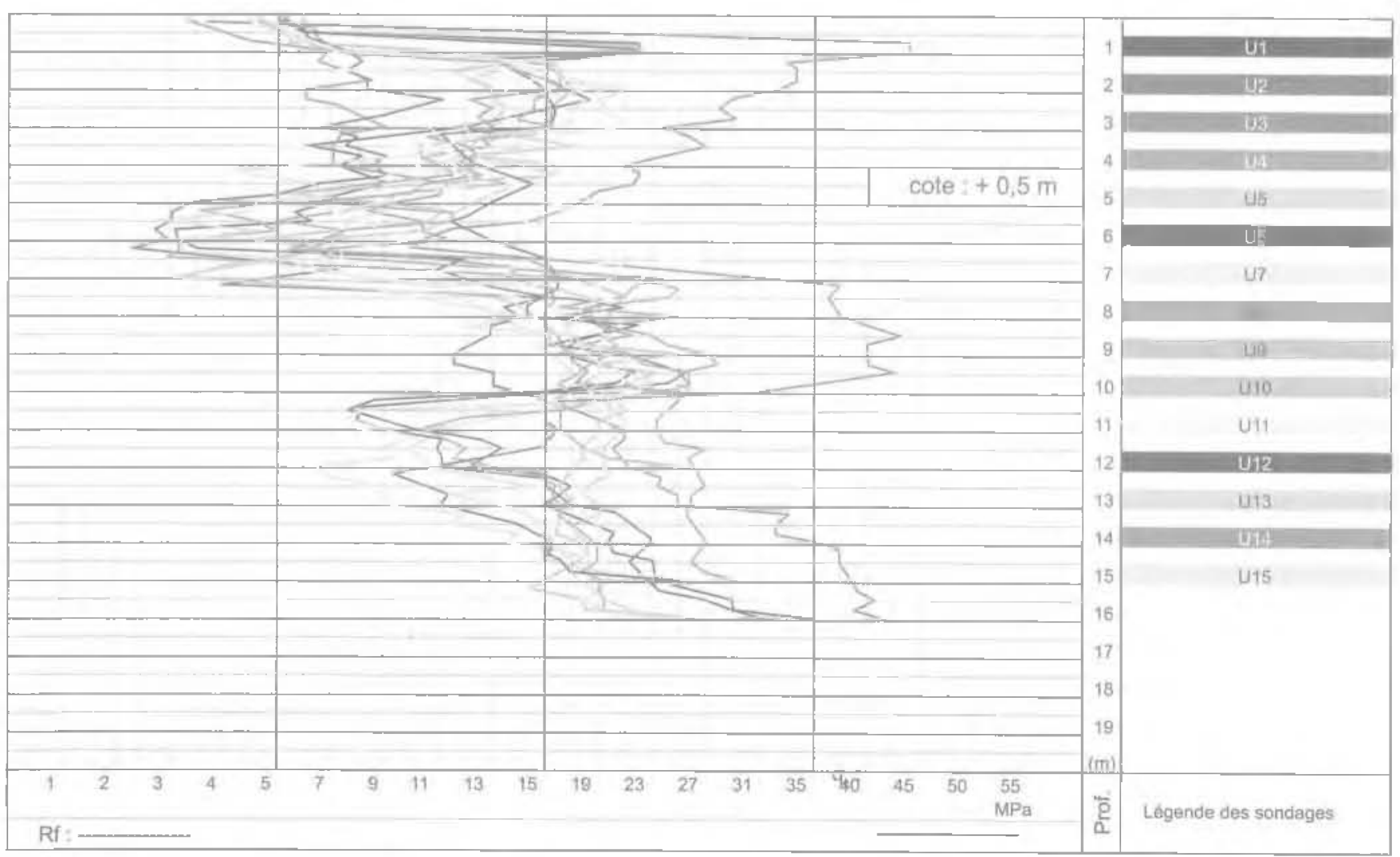

F16. 16 Résultats regroupés de 15 sondages effectués sur la parcelle 766, quarante-six mois après le traitement (U.1 à U.15).

Joined results of 15 soundings realized on plot 766, forty-six months after treatment (UW.1 to U.15). 
en 2004 , partant des coordonnées des repères pocés en 2002, a montré que le tassement tota! variait entre 14 et $30 \mathrm{~mm}$. c'est-à-dire de l'ordre du tassement prévu par les calculs.

\section{6}

\section{Conclusion}

L'amélioration obligatoire du remblai sableux hydraulique et du toutvenant obtenue en combinant les techniques du vibrocompactage et du piloriage intensif a permis d'aboutir à un tassement volsin de 1,5 m pout ce remblai ayant $8 \mathrm{~m}$ d'épaisseur naximum, c'est-à-dire une réduction de volume de l'ordre de $20 \%$.
Cette réduction immortante s'est tranuito par une augmentation notable de la résistance de pointe et du module de déformation, empèchant de ce fait tout risque de liquéfaction pour une secousse sismique caractérisée par le rapport $\mathrm{a} / \mathrm{g}=0,2$, valeur choisie compte tenu du contexte local.

Deux immeubles de douze étages fondés sur radier et construits sur ce sol amélioré ont accusé un tas sement mesuré au plus égal à $30 \mathrm{~mm}$, en très bonne concordance avec le résultat d"un calcul pressiomé. trique, les modules de déformation Ménard étant obtenus par corrélation à partir des valeur's de résis tance de pointe q mesurées au pénétromètre statique ANDINA.

\section{Bibliographie}

CEGP rCentre d'études géotechniques de Paris) - Règles d'utilisation des techniques pressiométriques et d'exploitation des résultats obtenus pour le calcul des fonclations. Brochure D/60/67. Techniques Loujs Mènard, 1968.

Corté J.-F. - Liquéfaction et essais de pénétration SPT. Bulletin de liaison des Laboratoires des Ponts et Chaussées $n^{\circ} 122$. 1982, p. 103-113.

CIRE (Comité technique régional européen! $n^{\circ} 4$ - Pressiomètres, utilisätion des résultats des essais pressio- métriques pour le dimensionmement des fondations en Europe; $11^{\text {re }}$ partie a Pressionètre Ménard/Pressionètre autoforeur i. A.A. Balkema, Rotterdam. 1991.

Davidovici V. - Gérie parasismique. Presses des Ponts et Chaussée. Paris, 1985.

Sanlaville P. - Etude géomorphologique de la région littorale du Liban. Tome II, Publications de l'Université libaraise. Section des études géographiques, Beyrouth. 1977 p. 602-606:

Seed H.B. Idriss I.M - Simplified procedure for evaluating soil liquefaction potential.
J. Soil Mechanics and Foundations DiviSion. ASCE, wol. 97, R. SM9, 1977.

Seed H.B.. Idriss I.M.. Arango I. - Evaluation of iquefaction potential of sand deposils based on observations of performance in previous earthquakes. J. Geothchn. Eng. Diw, ASCE, vol. 109, $n^{\circ} 3,1983$, p. $458-484$.

Seeg $\mathrm{H}$ B. Mori $\mathrm{K}$. Chan C K - Influence of seismic history on the liquefaction characteristics of sands. Report $n^{\circ}$ EERC $75-25$. Earthquake Engineering Research Cernter, University of Cailfornia, Berkeley, 1975. 